\title{
Power Distribution System Planning Evaluation by a Fuzzy Multi-Criteria Group Decision Support System
}

\author{
Tiefeng ZHANG \\ School of Electric\&Electronic Engineering, North China Electric Power University \\ Yonghua North Road, 619, Baoding, Hebei, 071003, P.R China \\ E-mail: ncepuztf@126.com \\ Guangquan ZHANG, Jun MA, Jie LU \\ Faculty of Engineering and Information Technology, University of Technology Sydney \\ PO BOX 123, Broadway, NSW 2007, Australia \\ E-mail: \{zhangg,junm,jielu\}@it.uts.edu.au
}

Received: 29-03-2010; Accepted: 25-05-2010

\begin{abstract}
The evaluation of solutions is an important phase in power distribution system planning (PDSP) which allows issues such as quality of supply, cost, social service and environmental implications to be considered and usually involves the judgments of a group of experts. The planning problem is thus suitable for the multi-criteria group decision-making (MCGDM) method. The evaluation process and evaluation criteria often involve uncertainties incorporated in quantitative analysis with crisp values and qualitative judgments with linguistic terms; therefore, fuzzy sets techniques are applied in this study. This paper proposes a fuzzy multi-criteria group decision-making (FMCGDM) method for PDSP evaluation and applies a fuzzy multi-criteria group decision support system (FMCGDSS) to support the evaluation task. We introduce a PDSP evaluation model, which has evaluation criteria within three levels, based on the characteristics of a power distribution system. A case-based example is performed on a test distribution network and demonstrates how all the problems in a PDSP evaluation are addressed using FMCGDSS. The results are acceptable to expert evaluators.
\end{abstract}

Keywords: Power distribution system planning, decision support systems, multi-criteria decision making, group decision-making, fuzzy sets.

\section{Introduction}

The evaluation of solutions is an important phase in power distribution system planning (PDSP), ${ }^{1}$ which consists of two stages. The first stage is to identify what characteristics must be incorporated by the power distribution system, what benefits the power distribution system will provide, and how consumers, the utility, and the government will be satisfied with the power distribution system. In the second stage, the decision mak- er(s) makes a decision on which planning solution will be determined and put into practice. Ranking planning solutions is the main task of the evaluation.

Traditionally, the solution that provides the optimum cost (i.e. provides the minimum economic and financial impact) would be selected for implementation after the corresponding power distribution system is assessed by performing network analysis when the power flows in a circuit or substation exceed designated capacities. $^{2-4}$ In today's demanding environment, more and more issues such as quality of supply, cost, social 
service and environmental implications must be considered in PDSP activities. ${ }^{1,5-7}$ At the same time, the planners are strongly involved in cost reduction projects to find the best compromise between performance and cost. Before implementation, the solutions should be evaluated by multi-criteria analysis of multi-aspects (technical, economic, environmental and social) by expert trade-off to determine the most suitable.

There are five issues which need to be considered to conduct PDSP evaluation.

(1) The determination of evaluation criteria and their degrees of importance to the requirements of the PDSP. In principle, PDSP evaluation is often very complex, because multiple evaluation criteria are hierarchical in structure, and these criteria have different roles in developing a solution. For PDSP, we need to consider criteria from technical, economic, environmental and social aspects. ${ }^{6}$ Multi-criteria decision making (MCDM) refers to making decisions for alternatives in the presence of multiple conflicting criteria. A main contribution area of MCDM is making a preference decision (e.g., evaluation, prioritization, selection) over the available alternatives, such as a set of alternatives that are characterized by multiple, usually conflicting, attributes. ${ }^{8}$ As PDSP involves multiple aspects and each aspect has a set of criteria and even sub-criteria, the PDSP evaluation needs a multi-level MCDM method to handle its hierarchical criteria system. In addition, some criteria may be more important than others, so criteria on all levels of an evaluation model need to be given individual weights.

(2) The PDSP evaluation requires the multiple perspectives of different people because one evaluator may not have enough knowledge to individually expertly assess an alternative. Therefore, evaluations of PDSP solutions are often made in groups, for example, by experts from different sections of electricity distribution companies and invited peer experts in this field, and require the gathering, processing and assessing of information from diverse functional groups within an organization. Group decision-making (GDM) is the process of arriving at a judgment or a solution for a decision problem based on the input and feedback of multiple individuals. However, the process may involve a set of uncertain factors, which includes an individual's role (weight) in the ranked planning solutions and an individual's preference and understanding of the planning solutions evaluated, and the criteria. ${ }^{9}$
(3) In PDSP evaluation, group members may have a different understanding of the same information, different experiences in the area of PDSP, and different preferences for different solutions. These different preferences among group members may impact directly on the solution evaluation results; therefore, the final ranking of solutions will be obtained by the suitable fusion and integration of these individuals' viewpoints. Because of the complexity, the fusion process has to be supported by a software tool.

(4) Both quantitative and qualitative criteria are included in PDSP evaluation, which require different techniques to handle them. Quantitative criteria are often indirect and are considered objective; thus, we call them indirect criteria. Qualitative criteria are often direct and being considered subjective, are called direct criteria. An indirect criterion is obtained by quantitative analysis (such as calculated by a physical or economical equation) with crisp values. A direct criterion is evaluated subjectively according to knowledge, obtained by qualitative judgments with linguistic terms. For linguistic terms, there is a requirement for linguistic information processing. For example, to express the weight of a criterion, the terms important and very important can be used; for an evaluator's weight, normal, important and more important can be used; and for a solution's score, linguistic term such as low and high could be used. The concept of linguistic variables is useful in dealing with situations that are too complex or ill-defined to be reasonably described in conventional quantitative expressions. ${ }^{10,11}$ Precise mathematical approaches are not sufficient to tackle such uncertain variables and derive a satisfactory solution.

(5) How to handle data uncertainty. In PDSP evaluation, cost coefficients, forecasting demand, electricity price, technology development and equipment failures are fraught with uncertainty, which will affect the data of indirect criteria. Even these linguistic terms reflect the uncertainty, inaccuracy and fuzziness of humans, and these uncertainties will affect the optimization process. Neglecting uncertainties in an optimization process may result in missed information and thus lead to non-robust decision support.

To deal with the above issues, many previous studies have formulated PDSP as an MCDM problem and proposed various methods. The simple multi-attribute rating technique (SMART) and analytical hierarchy process (AHP) ${ }^{12}$ were used to determine the weighted decision scores for each of the alternatives; a uniform 
possibility distribution was used to represent uncertain data entries. ${ }^{7}$ In Ref. 13, an interval AHP-based method was proposed and applied to a practical planning case, in which the uncertain information was handled by intervals. A fuzzy AHP-based method was proposed and applied in urban power system planning, ${ }^{14}$ in which fuzzy number was used to deal with data uncertainty. Outranking relation based techniques ${ }^{15,}{ }^{16}$ have been used for PDSP evaluation, and the uncertainty of data was incorporated by thresholds reflecting the decision maker's risk attitudes. Other MCDM methods used for PDSP evaluation include data envelopment analysis, ${ }^{17}$ grey correlation degree and TOPSIS ${ }^{18}$ and linear programming. ${ }^{16,19}$

Generally speaking, AHP is a most popular method used in PDSP evaluation, and to deal with the uncertainty of data, interval technology, ${ }^{13,19}$ fuzzy numbers ${ }^{14}$, ${ }^{20}$, probability distribution ${ }^{7}$ and outranking relation ${ }^{15,16}$ were employed in different situations. Each has its individual advantages and limitations.

At present, no methods have been used to deal with all five issues in PDSP evaluation, especially for the GDM aspect. Therefore, this paper proposes a fuzzy multi-criteria group decision-making (FMCGDM) method and applies a fuzzy multi-criteria group decision support system (FMCGDSS) to support the evaluation task.

This paper is organized as follows. Following the introduction, Section 2 presents a PDSP evaluation model. Section 3 describes a FMCGDM method. A FMCGDSS and its application in PDSP evaluation are illustrated by a PDSP case study in Section 4. Conclusions are outlined in Section 5.

\section{A PDSP Evaluation Model}

In this section, we analyze how the various factors and criteria interact and influence PDSP evaluation, and then present an evaluation model and its establishment process.

The planning of a power distribution system comprises development, operation and maintenance of the infrastructure as well as the integration of distributed generations and multiple end-uses. Objectives and tasks in the planning process may be summarized as:

- to cover supply duties with acceptable quality of supply and to contribute to effective power markets

- to specify infrastructure and power lines and substations at minimum cost, acceptable environmental impact, and good social services
The overall objective of PDSP evaluation can be formulated as "Maximizing society's welfare", and can be broken down naturally into four main major objectives, as in the evaluation model shown in Fig.1.

The evaluation model has three levels. The criteria on the first level are called aspects; the criteria on the

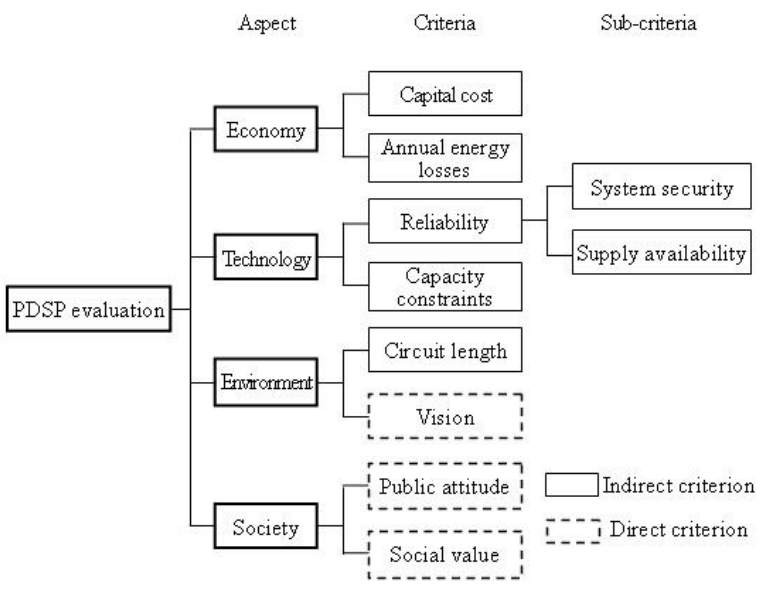

Fig. 1. A PDSP evaluation model

second are called criteria; and on the third level are called sub-criteria. These aspects and criteria (subcriteria) are determined by a group of experts in PDSP design by considering power distribution system characteristics and may be obtained in a variety of different ways, i.e. by economic analysis, judgmental assessment and technical analysis. These direct criteria can not be measured and easily quantified.

This evaluation model considers four aspects: 'Economy', 'Technology', 'Environment' and 'Society'. Each aspect has a set of criteria. For example, 'Technology' is assessed by two criteria, 'Reliability' and 'Capacity constraints'. Each criterion may be assessed by a set of sub-criteria; thus, the criterion 'Reliability' has two sub-criteria: 'System security' and 'Supply availability'. Evaluators need to give their evaluation (scores) on all these indirect criteria.

\subsection{Economy}

The 'Economy' aspect is probably the most important for the majority of decision makers. To assess this criterion, there are several objectives that can be considered separately, depending on who the decision maker is and the nature of his/her decision environment.

In this model, the 'Economy' aspect consists of 'Capital cost' and 'Annual energy losses'. 'Capital 
cost' is an important factor when assessing planning solutions, as it is a summation of the costs involved in implementing each of the options selected for each identified problem, plus any ongoing costs related to implementation or other operational aspects associated with the distribution network. The cost of each option (and solution) should be expressed either as the current cost of implementation, or as the future-worth equivalent at the end of the planning period (horizon year), converted using a present-worth calculation. The aim of the 'Annual energy losses' criterion is to provide an accurate assessment of the power losses associated with each planning solution. Here only real power losses associated with each solution will be calculated.

\subsection{Technology}

In this model, the 'Technology' aspect consists of the criteria 'Reliability', and 'Capacity constraints'. ${ }^{1}$ The criteria 'Reliability' consists of two sub-criteria: 'System security' and 'Supply availability'. Distribution network reliability is an issue of particular importance to large industrial connected customers, as even short supply interruptions may result in significant downtime and associated cost penalties in some countries.

'System security' is calculated as: number of customers interrupted per 100 connected customers = the sum of the number of customers interrupted for all incidents*100 / the number of connected customers.

'Supply availability' is calculated as: average customer minutes lost (CML) per connected customer = the sum of the customer minutes lost for all restoration stages for all incidents / the number of connected customers.

The criterion 'Capacity constraints' assesses each planning solution to identify where the system capacity remains below the forecasted scenario load, and it can be determined by performing a power flow calculation for each feasible solution for the load scenario.

\subsection{Environment}

Environmental impact is an important issue that must be considered by all distribution companies before the implementation of a PDSP project. Environmental impact includes land occupation, noise, aesthetic impact, and so on. Ideally, all impacts on nature, i.e. the whole lifecycle impact (construction, operation and disposal) of the various alternatives, should be included in the analysis.
Different solutions of a PDSP project have a different impact on the environment. The decision maker must decide which environmental aspects to take into consideration, according to the information about possible technical alternatives. Some types of environmental impact may be quantifiable while others will have to be considered in qualitative terms.

In this model, the 'Environment' aspect consists of two sub-criteria: 'Circuit length' and 'Vision'.

'Circuit length'1 is adopted to express the land occupation and to consider the total circuit length of new or modified network circuits, so it is quantifiable.

'Vision' ${ }^{6}$ is used to assess visual obstruction and mainly refers to the coordination of surrounding building or environment; it will be considered in linguistic terms.

Their degree of importance depends on the planning area. In an urban area or park, more attention will be paid to environmental impact than in a rural area.

\subsection{Society}

The main reason for building energy facilities and infrastructure is to provide society with energy services.

Increasing numbers of stakeholders with different objectives and criteria are involved in the planning process, such as electricity distribution companies, large-scale customers, regulators/local authorities, and local residents.

Some are directly involved as decision makers, while others are mainly affected by the final outcome without having taken an active part in the decision process. Therefore, it is useful to understand the social impact of various changes (local employment opportunities, local industrial development and potential public protests, etc.) in the power distribution system's infrastructure by taking into consideration social values and public attitudes in the planning process.

In this model, the 'Society' aspect includes 'Public attitude' and 'Social value'. 'Public attitude' is to minimize public protest and 'Social value' is to maximize service. It is not easy to measure public attitudes and social values, however, they are both direct criteria, and can be expressed by linguistic terms.

In this study, to allow each possible planning solution to be assessed, a PDSP evaluation model is established and several criteria have been selected. Nevertheless, this does not restrict the inclusion of additional criteria, if required, for particular planning studies. 


\section{A Fuzzy Multi-level Multi-Criteria Group Decision-Making Method for PDSP evaluation}

In this section, a fuzzy (multi-level) multi-criteria group decision-making (FMCGDM) method for PDSP evaluation is described. The FMCGDM method is based Zadeh's fuzzy set theory into conventional decisionmaking models ${ }^{21}$ and the concept of linguistic variables proposed by Zadeh ${ }^{22}$ to handle linguistic terms in a decision-making problem. In this method, AHP and TOPSIS $^{23}$ techniques are used and some of the GDM techniques ${ }^{24-29,31}$ are also incorporated.

For brevity, we introduce only the principle and steps of the proposed FMCGDM method below; some basic notions in FMCGDM method have been described in Ref. 30, and all fuzzy numbers are symmetric triangular numbers. $^{21}$

Stage one: Determine solutions, assessment-criteria, and individual weights, generate data for quantifiable criteria

Step 1: A set of solutions $S=\left\{S_{1}, S_{2}, \ldots, S_{m}\right\}$ is determined as alternatives and a set of decision makers (evaluators) $P=\left\{P_{1}, P_{2}, \ldots, P_{n}\right\}$ set up an evaluation group, generally $m, n \geq 2$. Also, three levels of assessment-criteria model within a tree hierarchy: $C=\left\{C_{1}, C_{2}\right.$, $\left.\ldots, C_{t}\right\}, C_{i}=\left\{C_{i 1}, C_{i 2}, \ldots, C_{i j_{i}}\right\}, i=1,2, \ldots, t$, and their sub-criteria $C_{i j}=\left\{C_{i j 1}, C_{i j 2}, \cdots, C_{i j k_{i j}}\right\}, j=1,2, \cdots, j_{i}$ for assessing these solutions are determined in the group, as shown in Fig.1. For those quantifiable criteria, data must be generated describing the performance of each solution in each criterion; those performance data are thought to be objective and will not be influenced by evaluators.

Step 2: Each evaluator is assigned a weight that is described by a linguistic term $\tilde{v}_{k}, k=1,2, \cdots, n$. These terms are determined through discussions in the evaluator group or assigned by a higher management level before, or at the beginning of, the decision process. Possible linguistic terms used here are Normal, Important, More important, and Most important, as shown in Table 1.

Step 3: Set up weights for all criteria within three levels. Let $W C=\left\{W C_{1}, W C_{2}, \ldots, W C_{t}\right\}, i=1,2, \ldots, t$ be the weights of criteria on level 1 , where $W C_{i}$ is described by a linguistic term. Possible linguistic terms used are as shown in Table 2.

For a criterion $C_{i}$, let $W C_{i}=\left\{W C_{i 1}, W C_{i 2}, \cdots, W C_{i j_{i}}\right\}, i=1,2, \ldots, t$ be the weights for the set of criteria on level 2 , and for a sub- criteria $\quad C_{i j}$, let $W C_{i j}=\left\{W C_{i j 1}, W C_{i j 2}, \cdots, W C_{i j k_{i j}}\right\}$, $j=1,2, \ldots, j_{i}$, be the weights for the set of criteria on level 3, where $W C_{i j}$ will be assigned a value from the same linguistic table as $W C_{i}$ above.

Table 1. Linguistic terms for describing weights of decision makers (evaluators)

\begin{tabular}{|l|c|}
\hline Linguistic terms & Fuzzy numbers \\
\hline Normal & $(2,3,4)$ \\
\hline Important & $(3,4,5)$ \\
\hline More important & $(4,5,6)$ \\
\hline Most important & $(5,6,6)$ \\
\hline
\end{tabular}

Table 2. Linguistic terms and related fuzzy numbers for describing the weights of aspects and criteria

\begin{tabular}{|l|c|}
\hline The importance degrees & Fuzzy numbers \\
\hline Absolutely unimportant & $(0,0,1)$ \\
\hline Unimportant & $(0,1,2)$ \\
\hline Less important & $(1,2,3)$ \\
\hline Important & $(2,3,4)$ \\
\hline More important & $(3,4,5)$ \\
\hline Strongly important & $(4,5,6)$ \\
\hline Absolutely important & $(5,6,6)$ \\
\hline
\end{tabular}

Stage two: Individual preference generation

Step 4: Set up the relevance degree of each alternative on each criterion for each evaluator $P_{y}$

Let $S C_{i j}^{y k}=\left\{S C_{i j 1}^{y k}, S C_{i j 2}^{y k}, \cdots, S C_{i j k_{i j}}^{y k}\right\}$ be the relevance degree of alternative $S_{k}$ on criterion $C_{i j}, i=1,2, \cdots, t, j=1,2, \cdots, j_{i}, k=1,2, \cdots, m$, where $S C_{i j k_{i j}}^{y k}$ is described by one of linguistic terms as shown in Table 3.

Table 3. Linguistic terms for preference of alternatives

\begin{tabular}{|l|c|}
\hline Linguistic terms & Fuzzy numbers \\
\hline Lowest & $(0,0,1)$ \\
\hline Very low & $(0,1,2)$ \\
\hline Low & $(1,2,3)$ \\
\hline Medium & $(2,3,4)$ \\
\hline High & $(3,4,5)$ \\
\hline Very High & $(4,5,6)$ \\
\hline Highest & $(5,6,6)$ \\
\hline
\end{tabular}

In this step, once the quantifiable criteria are maximized, the criteria data are mapped to a closed interval $[0,6]$. For each criterion, Let $M A X$ be the maximum value of alternatives, $M I N$ is the minimum value of alternatives, $M A X \neq M I N$, the alternative values are mapped to corresponding values in $[0,6]$ by a linear function described by 


$$
f(x)=\frac{6 *(x-M I N)}{M A X-M I N}
$$

The mapped values are then transformed to fuzzy numbers according to the symmetric triangular membership function as shown in Fig.2. For each criterion, $M A X$ is transformed to highest with the grade of 1 and $M I N$ is transformed to lowest with the grade of 1 , and each alternative value is transformed to the corresponding fuzzy number if the membership grade is the biggest. Here, if the biggest membership grade involves more than one fuzzy number, we adopt the right-hand one. As the criteria data is objective, all evaluators take the same transformed values as their individual preferences.

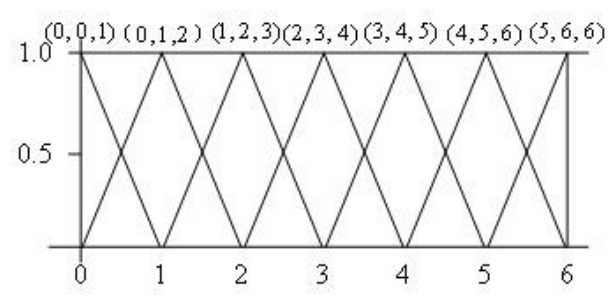

Fig.2. Symmetric triangular membership function

Step 5: Calculate the relevance degrees

The relevance degree $C S_{i}^{y k}$ of the aspect $C_{i}$ on the solutions $S_{k}, i=1,2, \ldots, t, k=1,2, \ldots, m$, are calculated by

$$
C S_{i}^{y k}=W C_{i} \times S C_{i}^{y k}=\sum_{j=1}^{j_{i}} W C_{i j} \times S C_{i j}^{y k}
$$

where $S C_{i j}^{y k}=\sum_{z=1}^{k_{i j}} W C_{i j z} \times S C_{i j z}^{y k}, i=1,2, \ldots, t, k=1,2, \ldots, m$.

Step 6: Calculate the aspect relevance degrees For the evaluator $P_{y}$, the relevance degree $S_{k}^{y}$ of the criteria $C$ on the alternatives $S_{k}, k=1,2, \ldots, m$ is calculated by using $S_{k}^{y}=C S^{y k} \times W C=\sum_{i=1}^{t} C S_{i}^{y k} \times W C_{i}$ $k=1,2, \ldots, m$. Here, $S_{k}^{y}$ is still a fuzzy number.

Step 7: Normalise the relevance degrees

The relevance degrees $S_{k}^{y} k=1,2, \ldots, m$

$$
\bar{S}_{k}^{y}=\frac{S_{k}^{y}}{\sum_{i=1}^{m} S_{k 0}^{y \mathrm{R}}}, \quad \text { for } k=1,2, \cdots, m .
$$

where the $S_{k 0}^{y \mathrm{R}}$ is the right end of 0-outset. ${ }^{9}$

Stage three: Group Aggregation

Step 8: Evaluator $P_{y}$ has already been assigned a weight that is described by a linguistic term $\tilde{v}_{y}, y=1,2, \cdots, n$ as shown in Table 1.

A weight vector is obtained:

$V=\left\{\tilde{v}_{y}, y=1,2, \cdots, n\right\}$.

The normalized weight of an evaluator $P_{y}(y=1,2$, $\ldots, n$ ) is denoted as

$$
\tilde{v}_{y}^{*}=\frac{\tilde{v}_{y}}{\sum_{i=1}^{n} v_{i 0}^{R}}, \text { for } y=1,2, \cdots, n .
$$

where the $v_{i 0}^{R}$ is the right end of 0-outset.

Step 9: Considering the normalized weights of all group members, we can construct a weighted normalized fuzzy decision vector

$$
\left(\tilde{r}_{1}, \tilde{r}_{2}, \cdots, \tilde{r}_{m}\right)=\left(\tilde{v}_{1}^{*}, \tilde{v}_{2}^{*}, \cdots, \tilde{v}_{n}^{*}\right)\left(\begin{array}{cccc}
\bar{S}_{1}^{1} & \bar{S}_{2}^{1} & \ldots & \bar{S}_{m}^{1} \\
\bar{S}_{1}^{2} & \bar{S}_{2}^{2} & \ldots & \bar{S}_{m}^{2} \\
\vdots & \vdots & \ddots & \vdots \\
\bar{S}_{1}^{n} & \bar{S}_{2}^{n} & \ldots & \bar{S}_{m}^{n}
\end{array}\right),
$$

where $\tilde{r}_{j}=\sum_{k=1}^{n} \tilde{v}_{k}^{*} \bar{S}_{j}^{k}$.

Step 10: In the weighted normalized fuzzy decision vector the elements $\tilde{r}_{j}, j=1,2, \cdots, m$, are normalized as positive fuzzy numbers and their ranges belong to the closed interval $[0,1] .{ }^{9}$ We can then define a fuzzy positive-ideal value $r^{*}$ and a fuzzy negative-ideal value $r$ as:

$$
r^{*}=1 \text { and } r^{-}=0 .
$$

The distances between each $\tilde{r}_{j}$ and $r^{*}, \tilde{r}_{j}$ and $r^{-}$can be calculated as:

$d_{j}^{*}=d\left(\tilde{r}_{j}, r^{*}\right) \quad$ and $\quad d_{j}^{-}=d\left(\tilde{r}_{j}, r^{-}\right), \quad j=1,2, \cdots, m,(6)$ where $d$ (.,.) is the distance measurement between two fuzzy numbers. ${ }^{9}$

Step 11: After the $d_{j}^{*}$ and $d_{j}^{-}$of each $S_{j}(j=1,2, \ldots$, $m$ ) are obtained, a closeness coefficient is defined to determine the ranking order of all solutions. The closeness coefficient of each alternative is calculated by ${ }^{9}$ :

$$
C C_{j}=\frac{1}{2}\left(d_{j}^{-}+\left(1-d_{j}^{*}\right)\right), \quad j=1,2, \cdots, m .
$$

The planning solution $S_{j}$ that corresponds to Max $\left(C C_{j}, j=1,2, \ldots, m\right)$ is the most suitable planning solution for the PDSP project.

This proposed FMCGDM method has been implemented in a FMCGDSS called Decider. ${ }^{30}$

\section{A Case Study using FMCGDSS for PDSP Evaluation}

The application of the proposed planning methodology to a test network based on an existing distribution network in an electricity distribution company is shown in Fig.3. ${ }^{1}$ 


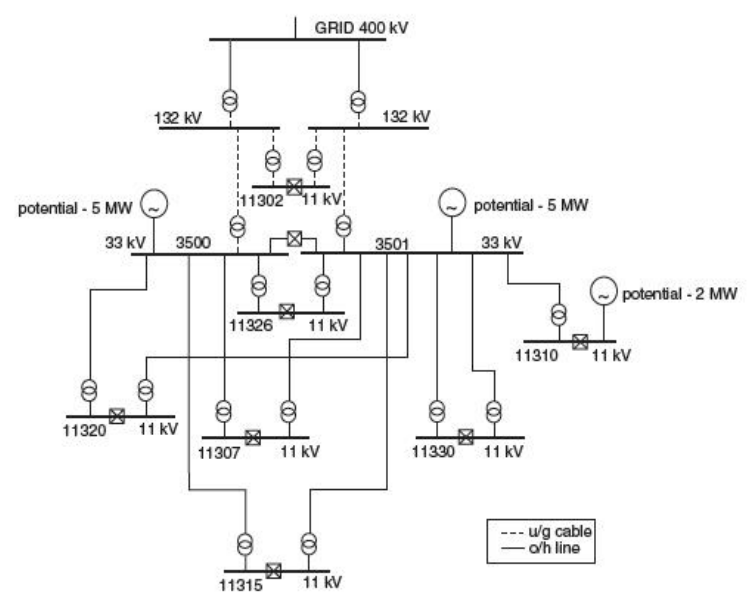

Fig.3. A test distribution network

The test network includes seven load centers, representing the accumulated load of the $11 \mathrm{kV}$ distribution network at each connection point, as well as 17 existing transformers and a number of existing underground cables and overhead lines. Owing to network load growth predicted for the planning period, load scenarios of $2.7 \%$ are considered. With the initial network data known, some relevant planning problems relating to the distribution network during the planning period are identified as follows: some transformers and circuits are overloaded at peak demand in the load scenario, some need replacement due to poor condition, and the $11 \mathrm{kV}$ load is currently experiencing poor reliability.

In our study, there are five different planning solutions to be evaluated by five evaluators based on the PDSP evaluation model shown in Fig.1.

The evaluators are five experts: a chief engineer as the leader of the group, a planner, a regulator, an expert representing large-scale customers and an invited peer expert. They are denoted as experts 1 to 5 respectively.

The data of indirect criteria have been obtained by network analysis and are shown in Table 4; they are minimized, so they will be processed to be maximized in calculation. The three remaining criteria are direct and will be determined by a group of experts/evaluators with linguistic terms; they are considered to be maximized in a positive direction.

Each of these aspects and criteria (sub-criteria) is assigned a weight, also determined by the group of experts. These weights reflect the importance degrees, influence degrees, and/or closeness of each criterion to its parent criterion. These weights are described in linguistic terms. For example, the criterion 'Reliability' can be very important to the aspect of 'Technology', so its weight is Absolutely important; the criterion 'Capacity constraints' can well represent the aspect 'Technology', and can thus have a weight of Strongly important. Table 5 shows the weights of these aspects and criteria corresponding to the model shown in Fig.1.

Table 4. The generated data of five solutions under the indirect criteria

\begin{tabular}{|c|c|c|c|c|c|}
\hline & Solution1 & Solution2 & Solution3 & Solution4 & Solution5 \\
\hline $\begin{array}{c}\text { Energy losses } \\
\text { MWh }\end{array}$ & 14632.43 & 14584.32 & 14657.32 & 14647.84 & 14674.76 \\
\hline System security & 4.72 & 4.69 & 4.42 & 4.93 & 4.66 \\
\hline Supply availability & 125.74 & 120.22 & 103.99 & 141.74 & 125.62 \\
\hline $\begin{array}{c}\text { Capacity constraint } \\
\text { MWh }\end{array}$ & 23.26 & 109.8 & 109.8 & 23.26 & 23.26 \\
\hline $\begin{array}{c}\text { Circuit length kM } \\
\text { Capital cost } \\
\text { £’000 }\end{array}$ & 1.44 & 1.59 & 2.36 & 0.68 & 1.44 \\
\hline
\end{tabular}

We also need to indicate that these weights can be changed when a PDSP project emphasizes a particular characteristic.

Each evaluator is assigned a weight that is determined by the group of experts through discussion and described by a linguistic term as shown in Table 6 .

For three direct criteria, evaluators are asked to provide scores such as Low, High, Very high and so on, for each solution. To obtain this data, a questionnaire, which has all the questions related to the three criteria described above, was designed to give to evaluators. In the questionnaire, almost all questions are designed in the form of statements, and the scores of the level of achievement of each planning solution was described in linguistic terms, as shown in Table 7. For example, if an evaluator agrees with the statement 'the planning solution looks very good for vision', he/she can give Very high (strongly agree) to the question (statement). This evaluation data will be entered into the FMCGDSS.

Table 5. The weights of aspects and criteria

\begin{tabular}{|c|c|c|}
\hline Aspects & Criteria & Sub-criteria \\
\hline \multirow{2}{*}{ Absolutely important } & \multicolumn{2}{|l|}{ Absolutely important } \\
\hline & \multicolumn{2}{|l|}{ More important } \\
\hline \multirow{3}{*}{ Absolutely important } & \multirow{2}{*}{ Absolutely important } & Absolutely important \\
\hline & & Absolutely important \\
\hline & \multicolumn{2}{|l|}{ Strongly important } \\
\hline \multirow{2}{*}{ More important } & \multicolumn{2}{|l|}{ Absolutely important } \\
\hline & \multicolumn{2}{|l|}{ Strongly important } \\
\hline \multirow{2}{*}{ Important } & \multicolumn{2}{|l|}{ More important } \\
\hline & \multicolumn{2}{|l|}{ More important } \\
\hline
\end{tabular}


Table 6. The weights of evaluators

\begin{tabular}{|c|c|}
\hline & Weight \\
\hline Expert 1 & Most important \\
\hline Expert 2 & Normal \\
\hline Expert 3 & More important \\
\hline Expert 4 & Important \\
\hline Expert 5 & More important \\
\hline
\end{tabular}

Table 7. The relevance degrees of solutions under direct criteria

\begin{tabular}{|c|c|c|c|}
\hline & Vision & Public attitude & Social value \\
\hline \multicolumn{4}{|l|}{ Expert 1} \\
\hline Solution 1 & Medium & High & Medium \\
\hline Solution 2 & Medium & Low & Medium \\
\hline Solution 3 & Very low & Very low & Medium \\
\hline Solution 4 & Very high & Highest & Very high \\
\hline Solution 5 & Very high & High & Very high \\
\hline \multicolumn{4}{|l|}{ Expert 2} \\
\hline Solution 1 & Medium & Medium & Medium \\
\hline Solution 2 & Medium & Medium & Medium \\
\hline Solution 3 & Low & Low & Low \\
\hline Solution 4 & Highest & Highest & High \\
\hline Solution 5 & High & Very high & Very high \\
\hline \multicolumn{4}{|l|}{ Expert 3} \\
\hline Solution 1 & Medium & Low & Medium \\
\hline Solution 2 & Medium & Medium & Medium \\
\hline Solution 3 & Very low & Low & Low \\
\hline Solution 4 & Highest & Highest & High \\
\hline Solution 5 & Very high & High & Very high \\
\hline \multicolumn{4}{|l|}{ Expert 4} \\
\hline \begin{tabular}{|l} 
Solution 1 \\
\end{tabular} & Low & Medium & Medium \\
\hline Solution 2 & Low & Low & Low \\
\hline Solution 3 & Lowest & Lowest & Medium \\
\hline Solution 4 & Highest & Highest & High \\
\hline Solution 5 & High & Very high & High \\
\hline \multicolumn{4}{|l|}{ Expert 5} \\
\hline Solution 1 & Medium & High & Medium \\
\hline Solution 2 & Low & Low & Low \\
\hline Solution 3 & Very low & Very low & Medium \\
\hline Solution 4 & Highest & Highest & Very high \\
\hline Solution 5 & Very high & High & Highest \\
\hline
\end{tabular}

The obtained objective value is then transformed into an integral numeric evaluation value between $(1,7)$ according to a pre-defined membership function. ${ }^{30}$

After obtaining weights (importance degrees) for these aspects and their criteria, and all scores for the five planning solutions, the group can use the newly developed FMCGDSS to conduct the performance evaluation results and ranking among the planning solutions evaluated.
The working process of the FMCGDSS is summarized in seven steps as follows:

Step 1: Create a new file for the evaluation issue (Fig.4, Fig.5).

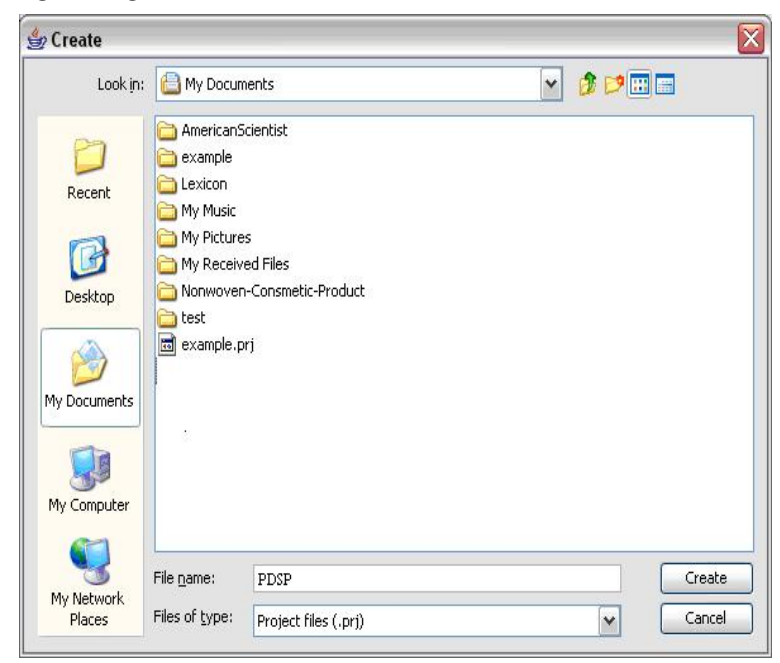

Fig.4. Creating a new project for evaluation

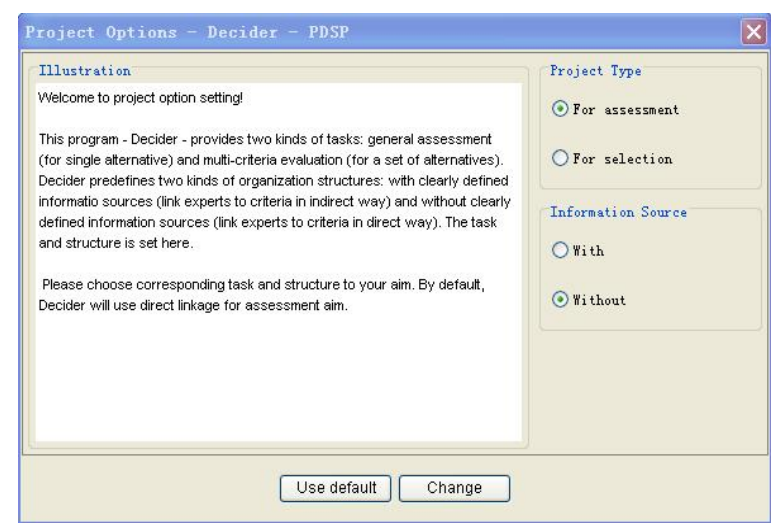

Fig.5. Setting up a PDSP evaluation problem

Step 2: Input the PDSP evaluation model with its levels of criteria, criteria weights, and related descriptions.

Fig.6 shows the PDSP model and a criterion's weight and description.

Fig.7 shows the data input process of a criterion. 


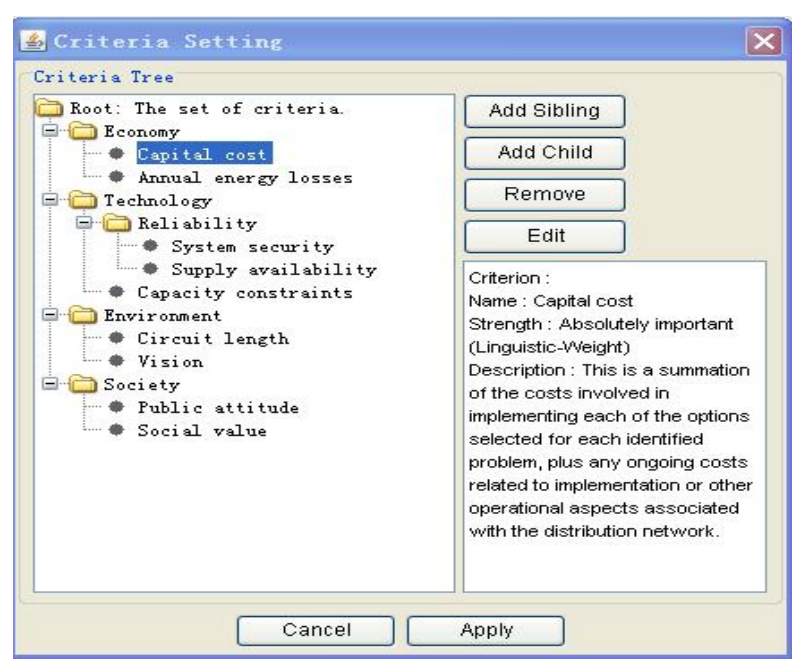

Fig.6. The evaluation model with three levels of criteria

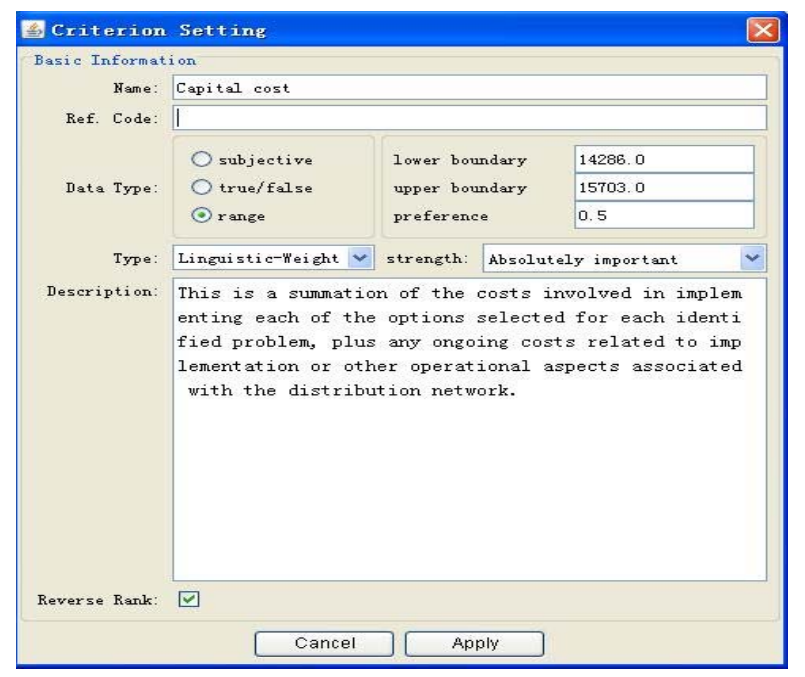

Fig.7. Data entry process of a criterion

Step 3: Input evaluators and their weights in linguistic terms.

Fig.8 shows the five evaluators, an evaluator's information and weights are shown in Fig.9.

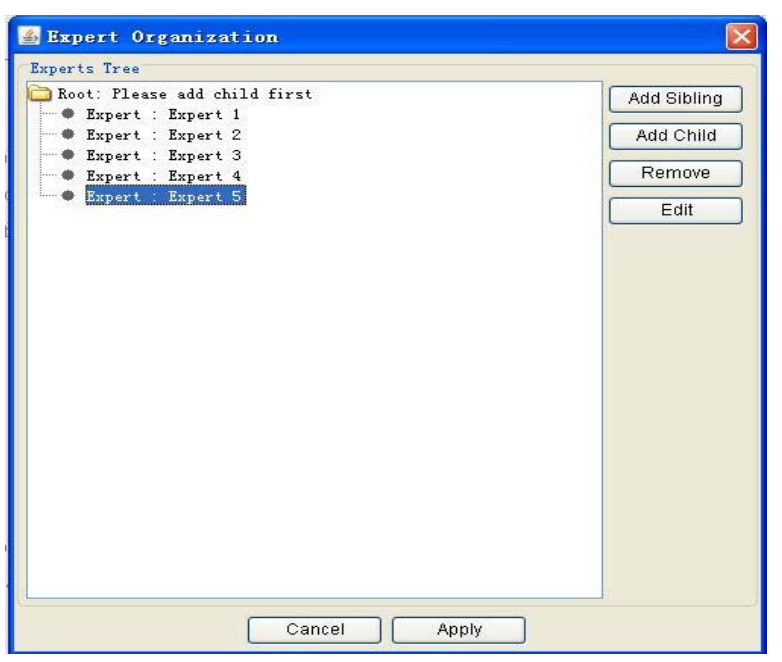

Fig.8. All members of the evaluation group are entered

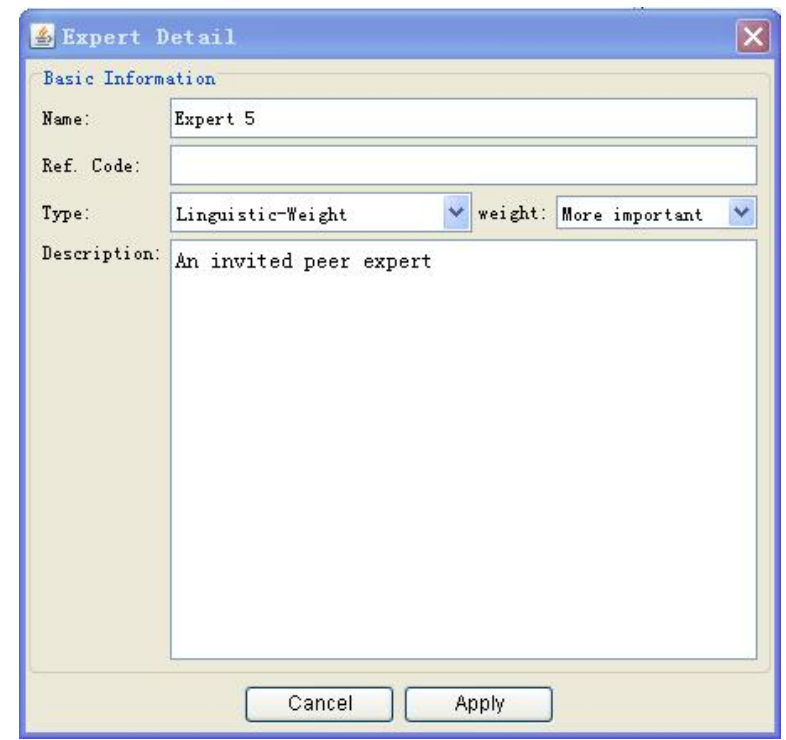

Fig.9. An evaluator's information and weights

Step 4: Input planning solutions to be evaluated. Fig.10 shows these solutions' names. 


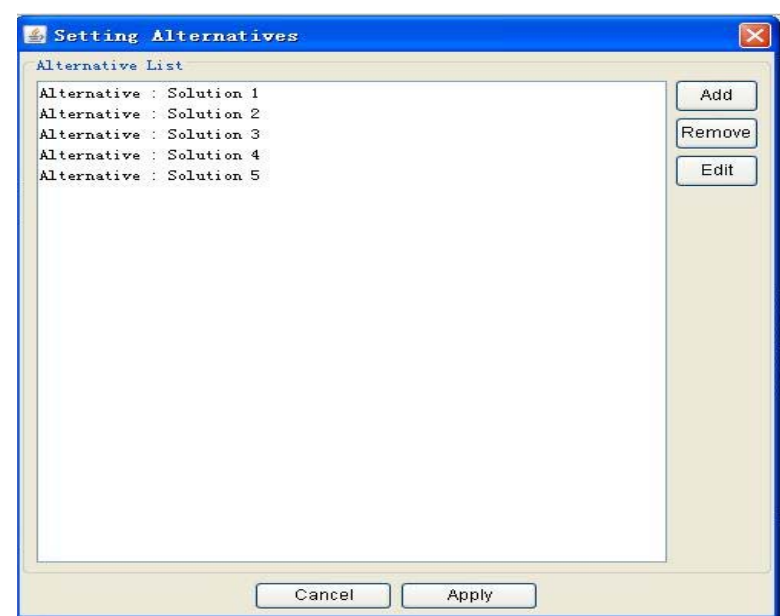

Fig.10. All planning alternatives to be evaluated

Step 5: Enter all the evaluator scores.

Based on the criteria, for each planning solution, every evaluator has a score to be entered (Fig.11).

\begin{tabular}{|c|c|c|c|c|c|c|}
\hline \multicolumn{7}{|c|}{ Assesspent Input } \\
\hline \multicolumn{7}{|l|}{ Assessments } \\
\hline Al ternative: & \multicolumn{3}{|c|}{ Alternative: Solution 4} & \multicolumn{3}{|c|}{ Add } \\
\hline Expert: & Expert & Expert 5 & $\checkmark$ & \multicolumn{3}{|c|}{ Remove } \\
\hline Criteria: & \multicolumn{3}{|c|}{ Social value--1 } & & & \\
\hline Assessment: & \multicolumn{3}{|c|}{ Very high } & & & \\
\hline Assessment Type: & \multicolumn{3}{|c|}{ Linguistic-Scores } & & & \\
\hline Sorutron 4 EX & xpert 4 & ruolic atritude & standara-scale & & 0 & - \\
\hline Solution 4 & xpert 5 & Public attitude & Standard-Scale & & 6 & \\
\hline Solution 4 & xpert 1 & Social value & Standard-Scale & 5 & & \\
\hline Solution 4 & xpert 2 & Social value & Standard-Scale & 4 & & \\
\hline Solution 4 & xpert 3 & Social value & Standard-Scale & 4 & & \\
\hline Solution 4 & xpert 4 & Social value & Standard-Scale & 4 & 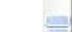 & \\
\hline Solution 4 & xpert 5 & Social value & Standard-Scale & 5 & 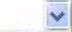 & 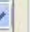 \\
\hline \multirow[t]{2}{*}{$\leqslant$} & & (IIII & & & $>$ & \\
\hline & & Vancel & apply & & & \\
\hline
\end{tabular}

Fig.11. Assessment input for all alternatives under all criteria lem.

Step 6: Generate the final ranking result of the prob-

When all data is entered in this software, we run the developed FMCGDM method. We can see that planning Solution 3 receives the highest closeness coefficient value and therefore is the most suitable solution for this PDSP project.

Fig.12 shows the group's evaluation result for these planning solutions. We can also conduct further analysis on the evaluation result in each aspect or each subgroup.

Fig.13 shows evaluation results on the criterion 'Vision' of the five solutions where Solution 4 has the highest score.

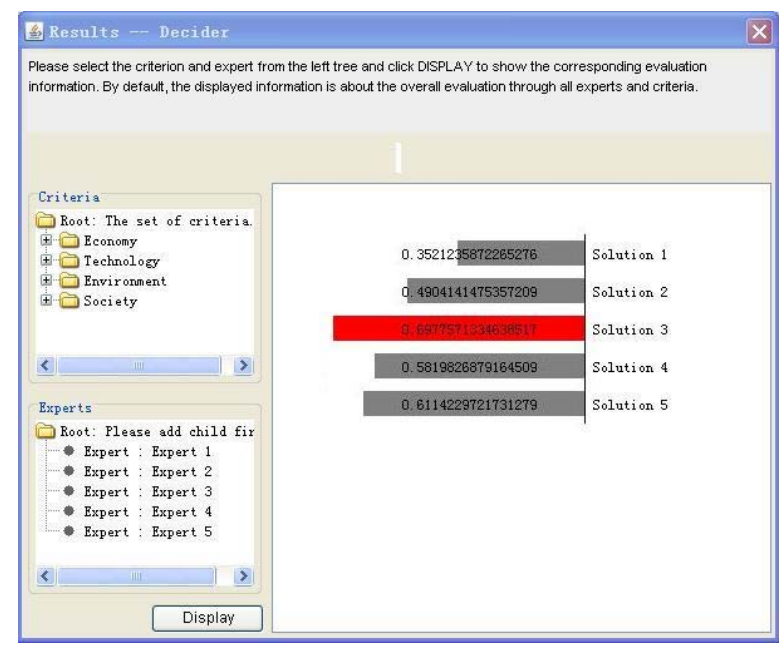

Fig.12. Final ranking result for possible planning solutions

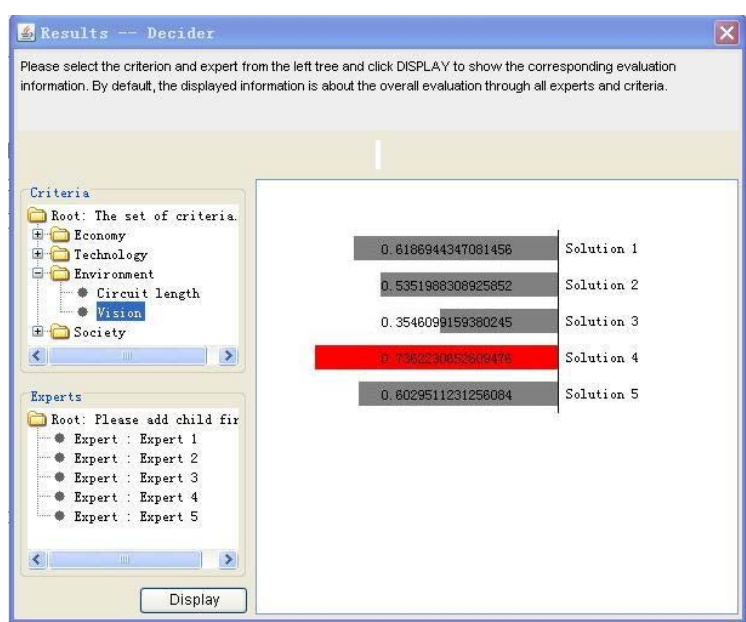

Fig.13. Ranking result for solutions under the criterion 'Vision'

The case study for PDSP evaluation shows that the proposed method can deal with all issues mentioned in this paper in a comprehensive way and expert evaluators are satisfied with the results and evaluation process.

\section{Conclusions}

The comprehensive evaluation of planning solutions is an important phase in the PDSP process to ensure the development of a robust planning solution. This evaluation process is a preference based decision but involves a complex situation in which both quantitative and qualitative criteria are within a hierarchy and multiple members are involved with different opinions. In particular, experts' opinions are often in vague, using crisp numbers to express is not sufficient. Therefore, a 
FMCGDM method is presented in this paper and applied to evaluate PDSP solutions.

The main advantage of our work from previous studies is that we not only formulate PDSP evaluation as an MCDM problem, but also take into account GDM for all evaluators' preferences. In particular, linguistic variables are used to express human judgment and fuzzy sets are applied to present and calculate linguistic variables. In practical projects of PDSP evaluation, the quantitative values of criteria are often difficult to determine and the situation is far more complex than that discussed in this paper. Using linguistic variables in evaluations is very common, and the evaluation process often involves an expert team, so the proposed FMCGDM method and the FMCGDSS are expected to have more real cases. Since experts have different professional knowledge backgrounds and practical experiences, the assigned weight for each expert should vary from criterion to criterion, rather than being the same for all criteria, as in this study, which will be improved in future work.

\section{Acknowledgements}

The work presented in this paper was partly supported by the Australian Research Council (ARC) under discovery project DP0880739.

\section{References}

1. P. Espie, G.W. Ault, G.M. Burt and J.R. McDonald, Multiple criteria decision making techniques applied to electricity distribution system planning, IEE ProceedingsGeneration, Transmission and Distribution, 150(5) (2003) 527-535.

2. T. Gonen and I.J. Ramirez-Rosado, Optimal multi-stage planning of power distribution systems, IEEE Trans. on Power Delivery, 2(2) (1987) 512-519.

3. Y. Tang, Power distribution system planning with reliability modeling and optimization, IEEE Trans. on Power Systems, 11(1) (1996) 181-189.

4. A. Chowdhury, T.C. Mielnik, L.E. Lawton, M.J. Sullivan and A. Katz, Reliability worth assessment in electric power delivery systems, Proceeding of the 2004 IEEE Power Engineering Society General Meeting (Denver, CO, United States, Jun 2004), Vol.1, pp. 654-660.

5. S. Wong, K. Bhattacharya and J.D. Fuller, Electric power distribution system design and planning in a deregulated environment, IET- Generation, Transmission \& Distribution, 3(12) (2009) 1061-1078.

6. E. Jordanger, B.H. Bakken, A.T. Holen, A. Helseth and A. Botterud, Energy distribution system planningmethodologies and tools for multi-criteria decision analysis, Proceeding of the 18th International Conference and
Exhibition on Electricity Distribution(Turin, Italy, 2005), pp.1-5.

7. P. Espie, G.W. Ault and J.R. McDonald, Multiple criteria decision making in distribution utility investment planning, Proceeding of the 2000 International Conference on Electric Utility Deregulation and Restructuring and Power Technologies (London, UK, 2000), pp.576-581.

8. K. Yoon and C. Hwang, Multiple attribute decision making - An introduction (SAGE Publications, Thousand Oaks, 1995).

9. J. Lu, G. Zhang, D. Ruan and F. Wu, Multi-objective group decision making: methods, software and applications with fuzzy set technology (Imperial College Press, London, 2007).

10. N. Karacapilidis and C. Pappis, Computer-supported collaborative argumentation and fuzzy similarity measures in multiple criteria decision-making, Computers and $\mathrm{Op}$ erations Research, 27(7) (2000) 653-671.

11. M. Marian, I. Hatono and H. Tamura, Linguistic labels for expressing fuzzy preference relations in fuzzy group decision making, IEEE Trans. on Systems, Man, and Cybernetics, 28(2)(1998)205-217.

12. T. L. Saaty, The analytic hierarchy process (McGrawHill, New York, 1980).

13. J. Xiao, C.S. Wang and M. Zhou, IAHP-based MADM method in urban power system planning, Proceedings of the Chinese Society of Electrical Engineering, 24(4) (2004) 50-57.

14. D.Y. Chen, J. Xiao and C.S. Wang, A FAHP-based MADM method in urban power system planning, Proceedings of Electric Power System and Automation, 15(4) (2003) 83-88.

15. T.F. Zhang and J.S. Yuan, Decision-aid for power distribution system planning problems using ELECTRE III, Proceeding of the 7th Power Engineering International Conference, (Singapore, Dec. 2005).

16. J.S. Yuan, T.F. Zhang, J.X. Liu and Y.H. Kong, An MADM method based on outranking relation and linear programming in power distribution system planning, Proceedings of the Chinese Society of Electrical Engineering, 26(12) (2006) 106-110.

17. G. Wei, W.L. Wu, J. Liu and X. Zhang, Comprehensive judgment for power system planning alternatives based on SE-DEA model, Power System Technology, 31(24) (2007) 12-16.

18. L.G. Fan and Y.H. Li , A new MCDM method in transmission network planning based on gray correlation degree and TOPSIS, Proceeding of the 27th Chinese Control Conference(Kunming, China, 2008), pp.462-467.

19. J. Pan, Y. Teklu and A. de Castro, An interval-based MADM approach to the identification of candidate alternatives in strategic resource planning, IEEE Trans. on Power Systems, 15(4) (2000) 1441-1446.

20. D.P. Bernardon, V.J. Garcia, A.S.Q. Ferreira, L.N. Canha and A.R. Abaide, New fuzzy multicriteria decision making algorithm to distribution network reconfiguration, Proceeding of the 44th Universities Power Engineering 
International Conference (Glasgow, Scotland. 2009), pp.1-5.

21. H. Zimmermann, Fuzzy sets, decision making and expert systems (Kluwer Academic Publishers, Dordecht, 1987).

22. L.A. Zadeh, The concept of a linguistic variable and its application to approximate reasoning-part 1, Information Sciences, 8(3) (1975) 199-249.

23. C.T. Chen, Extensions of the TOPSIS for group decision making under fuzzy environment, Fuzzy Sets and Systems, 114(1) (2000) 1-9.

24. U. Bose, A.M. Davey and D.L. Olson, Multi-attribute utility methods in group decision making: past applications and potential for inclusion in GDSS, Omega, 25(6) (1997) 691-706.

25. G. Pasi and R.R. Yager, Modeling the concept of majority opinion in group decision making, Information Sciences, 176(4) (2006) 390-414.

26. J. Kacprzyk, M. Fedrizzi and H. Nurmi, Group decision making and consensus under fuzzy preference and fuzzy majority, Fuzzy Sets and Systems, 49(1) (1992) 21-31.

27. H. Lee, Group decision-making using fuzzy set theory for evaluating the rate of aggregative risk in software development, Fuzzy Sets and Systems, 80(3) (1996) 261-271.

28. J. Wang and Y. Lin, A fuzzy multicriteria group decision making approach to select configuration items for software development, Fuzzy Sets and Systems, 134(3) (2003) 343-363.

29. G. Zhang and J. Lu, An integrated group decision making method with fuzzy preference for alternatives and individual judgments for selection criteria, Group Decision and Negotiation, 12(6) (2003) 501-515.

30. J. Ma, J. Lu and G. Zhang, Decider: a fuzzy multi-criteria group decision support system, Knowledge-Based Systems, 23(1) (2010) 23-31.

31. J. Lu, G. Zhang and F. Wu (2008), Team situation awareness using web-based fuzzy group decision support systems, International Journal on Computational Intelligence Systems 1(1) (2008) 51-60. 